\title{
WIRELESS DATA TRANSMISSION THROUGH UART PORT USING ARM \& RF TRANSCEIVER
}

\author{
J.K.Singh ${ }^{1}$, Atul Srivastava ${ }^{2}$, Rahul S.Parbhane ${ }^{3}$ \\ ${ }^{1}$ Asst Professor, Department of E\&TC, VACOE, Ahmednagar \\ ${ }^{2}$ Asst Professor, Department of E\&TC, VACOE, Ahmednagar \\ ${ }^{3}$ Sr. Lecturer Department of E\&TC, VACOE, Ahmednagar
}

\begin{abstract}
This paper presents the simulation and test result of a project for Wireless Data transmission through UART Port using ARM \& RF Transceiver in license free band of frequencies $2.4 \mathrm{GHz}$ to $2.4835 \mathrm{GHz}$. It uses ARM7 and MRF24J40 for Wireless Data transmission through UART Port for short-range up to 500 meters. The speed of data transmission is of $250 \mathrm{kbps}$. The Output power of source module for 500 meters is $0 \mathrm{dBm}$ to $22 \mathrm{dBm}$. The Receiver sensitivity of the receiver module is $-95 \mathrm{dBm}$
\end{abstract}

Keywords- ARM (Advance Reduced Machines), UART (Universal Asynchronous Receiver Transmitter), ISM (Industrial Scientific and Medical), SPI (Serial Peripheral Interface)

\section{INTRODUCTION}

In this paper ISM band is used for wireless in license free band of frequencies $2.4 \mathrm{GHz}$ to $2.4835 \mathrm{GHz}$. It uses ARM7 for management and control of data communication for short-range up to 500 meters and long range up to $10 \mathrm{~km}$. This paper present a designed for short range application. It incorporates two main IC module i.e., ARM controller[1] and RF Transceiver IC[2]; one is used for controlling and monitoring the data communication and other is used for transmitting and receiving the data with antenna into free space for short range.

\section{UNIVERSAL ASYNCHRONOUS RECEIVER/}

\section{TRANSMITTER}

In this project the programming is done for RS232 interface. LPC2378 controller IC has four UART ports. It is used for serial data communication between the ports and interfaces like RS232, RS 485/422.

\subsection{Features}

a) 16 byte Receive and Transmit FIFOs.

b) Register locations conform to ' 550 industry standard.

c) Receivers FIFO trigger points at 1, 4, 8, and 14 bytes.

d) Built-in baud rate generator.

e) Fractional divider for baud rate control, auto baud capabilities, and implementation of software or hardware flow control.

f) UART3 includes an IrDA mode to support infrared communication.

\section{SERIAL PERIPHERAL INTERFACE (SPI)}

The SPI port is used for interfacing the controller IC to RF $\mathrm{IC}[2]$. It is used for the transferring the data serially through
MOSI and MISO. It is having six pins i.e SCK, MISO, MOSI, RESET, SSEL and WAKE pin.

SPI is a full duplex serial interfaces. It can handle multiple masters and slaves being connected to a given bus. Only a single master and a single slave can communicate on the interface during a given data transfer. During a data transfer the master always sends 8 to 16 bits of data to the slave, and the slave always sends a byte of data to the master.

\subsection{Features}

a) Compliant with Serial Peripheral Interface (SPI) specification.

b) Synchronous, Serial, Full Duplex Communication.

c) SPI master or slave.

d) Maximum data bit rate of one eighth of the input clock rate.

e) 8 to 16 bits per transfer.

\section{RF TRANSCEIVER}

The RF Transceiver is a low-cost, low-power, low data rate (250 or $625 \mathrm{kbps}$ ) Wireless Personal Area Network (WPAN) device. The project module is using MRF24J40 IC for this RF Transceiver.

Features:

a) IEEE 802.15.4 Standard Compliant RF Transceiver

b) Supports ZigBe, MiWi, MiWi P2P and Proprietary Wireless Networking Protocols

c) Simple, 4-Wire Serial Peripheral Interface (SPI)

d) Integrated $20 \mathrm{MHz}$ and $32.768 \mathrm{kHz}$ Crystal Oscillator Circuitry

e) ISM Band 2.405-2.48 GHz Operation

f) Data Rate: $250 \mathrm{kbps}$ (IEEE 802.15.4); $625 \mathrm{kbps}$ (Turbo mode)

g) $\quad-95 \mathrm{dBm}$ Typical Sensitivity with $+5 \mathrm{dBm}$ Maximum Input Level 
h) $\quad+0 \mathrm{dBm}$ Typical Output Power with $36 \mathrm{~dB}$ TX Power Control Range

\section{BLOCK DIAGRAM OF PROJECT MODULE}



Fig. 1: Block Diagram of wireless ISM Transceiver

Supporting Features

a) It supports the user interfaces like RS-232, RS485/RS-422, Ethernet and USB.

b) RF interface: Antenna port.

c) RF output power: $0 \mathrm{dbm}$ to $+28 \mathrm{dbm}$.

d) Data rate (RF transceiver): $250 \mathrm{Kbps}$.

e) Supports both indoor and outdoor application.

f) Supports 802.15.4 MAC standard.

g) Communication range for outdoor application is up to $15 \mathrm{Km}$

\section{SNAPSHOT OF PROJECT MODULE}

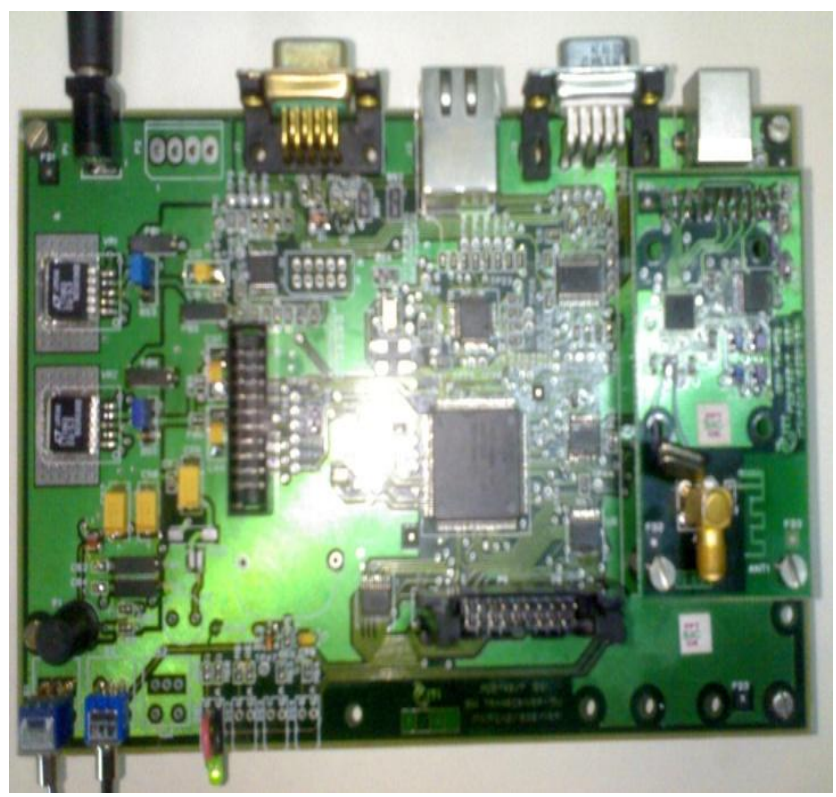

Fig. 2: Snapshot of project module

\section{PROGRAMMING OF RS-232 INTERFACE}

The programming of ARM controller (LPC2378) is done on Kiel software tool. This tool uses C- language for writing the program. All the necessary control registers of ARM Controller IC and RF IC is configured in the program. Before writing the Algorithm for the programming of RS232 interface, the data flow diagram should be analyzed.

\subsection{Algorithm for Data Transmission using RS-232}

\section{Interface}

\section{Steps:}

1. Initialize ARM7 CONTROLLER IC LPC2378.

2. Initialize RF IC- MRF24J40.

3. Configure the UART port0 for RS232 in TX/RX mode.

4. Configure the SPI of LPC2378.

5. Configure the SPI of RF IC by enabling CS complement pin.

6. If station 1 is made as master; then configure RF IC in non beacon TX mode.

7. If station 1 is made as slave, then configure RF IC in non beacon RX mode.

8. If station 1 is as master; then perform the following operations:

(a) Receive the data from serial UART port0 (RS232).

(b) Read UORBR registers of ARM controller.

(c) Write or copy the data from UORBR to SOSPDR.

(d) Copy the data from SOSPDR to TXnFIFO register of RF IC.

9. Put the loop for the step-8 for continuous data transmission.

\subsection{Algorithm for Data Reception Using Rs-232}

\section{Interface}

\section{Steps:}

1. Initialize ARM7 CONTROLLER IC LPC2378.

2. Initialize RF IC- MRF24J40.

3. Configure the UART port0 for RS232 in TX/RX mode.

4. Configure the SPI of LPC2378.

5. Configure the SPI of RF IC by enabling CS complement pin.

6. If station 1 is made as master; then use TX mode algorithm.

7. If station 1 is made as slave, then configure RF IC in nonbeacon RX mode and perform following operations:

(a)Receive the data from RF Antenna.

(b) Read the data from RXnFIFO register.

(c) Copy the data from RXnFIFO to SOSPDR of ARM IC.

(d) Write or copy the data SOSPDR to UnTHR of UART port.

8. Put the loop for the step-7 for continuous data reception. 


\section{FLOWCHART FOR UART PORT}

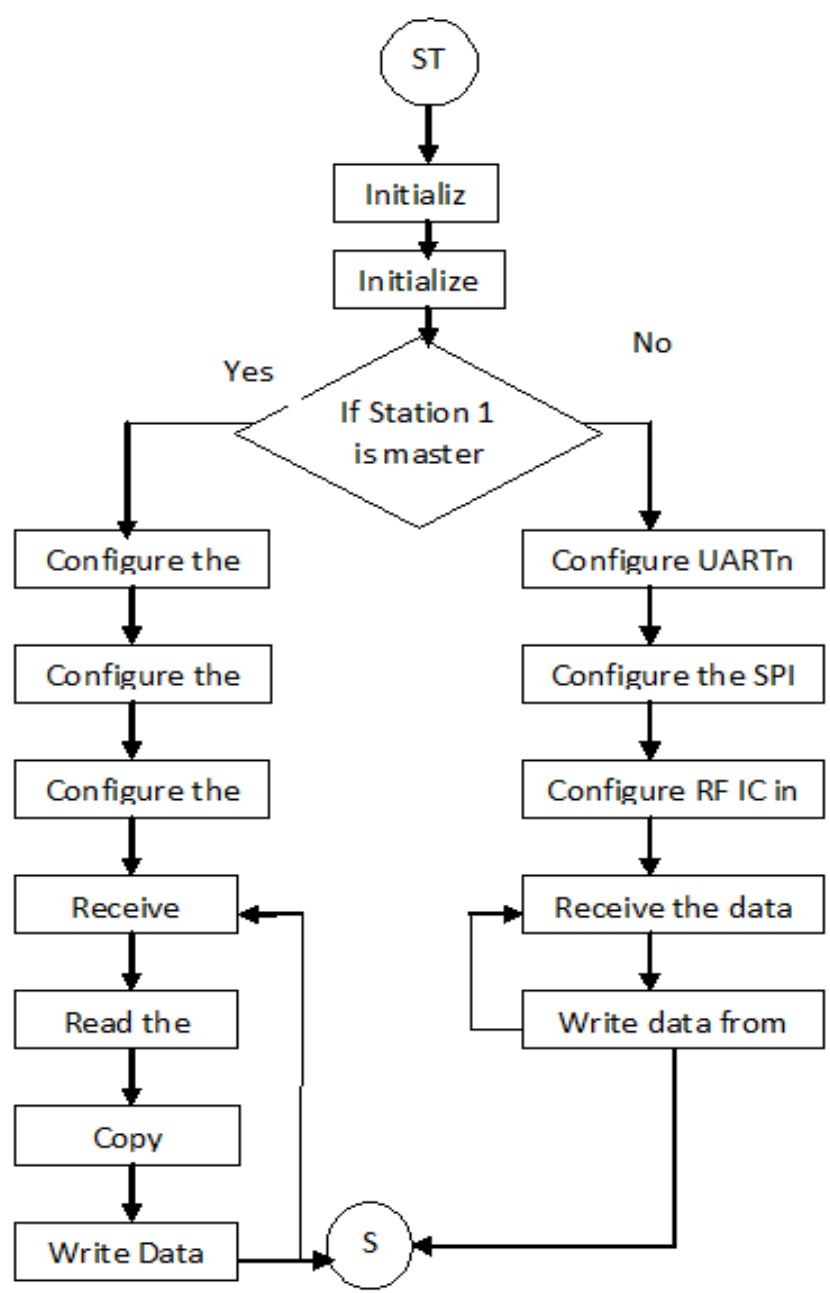

Fig. 3: Flowchart for UART port

\section{RESULTS AND DISCUSSIONS}

There are different test methods to verify the working of the project module. These are given in the table. 1 and the corresponding test results are given in the next sections.

Test Methods:

(a) Received signal strength in $\mathrm{dBm}$ for different ranges.

(b) Hypertext mode for serial wireless data communication with RS-232 interface by HyperTerminal of Personal computer.

(c) Continuous data transmission at MOSI (master out slave

in) of SPI port with SCK clock.

(d) Test of Program by debugging and its Performance analyses.

\subsection{Test Results for Received Signal Strength in} dBm for Different Ranges

The following table. 1 shows the different reading taken between the two ISM transceiver module i.e source module and receiver module during the continuous serial data transmission for different indoor application.
Table.1: Received level observed for indoor applications.

\begin{tabular}{|l|l|l|l|l|}
\hline $\begin{array}{l}\text { S1 } \\
\text { No. }\end{array}$ & $\begin{array}{l}\text { Source } \\
\text { Frequency } \\
(\mathrm{GHz})\end{array}$ & $\begin{array}{l}\text { Distance } \\
(\text { metre })\end{array}$ & $\begin{array}{l}\text { Source } \\
\text { Feed } \\
\text { level } \\
(\mathrm{dBm})\end{array}$ & $\begin{array}{l}\text { Destination } \\
\text { Receive } \\
\text { level }(\mathrm{dBm})\end{array}$ \\
\hline 1. & 2.45 & 1 & 0 & -52 \\
\hline 2. & 2.45 & 2.5 & 0 & -57 \\
\hline 3. & 2.45 & 15 & 0 & -72 \\
\hline 4. & 2.45 & 35 & 0 & -77 \\
\hline 5. & 2.45 & 75 & 0 & -82 \\
\hline 6. & 2.45 & 150 & 0 & -88 \\
\hline 7. & 2.45 & 300 & 0 & -94 \\
\hline
\end{tabular}

The RSSI value is an 8-bit value ranging from $0-255$. The mapping between the RSSI values with the received power level is shown in below figure.



Fig. 4: RSSI vs. RECEIVED POWER (dBM)

9.2 Snapshots of Test Results for Hypertext Mode for Serial Wireless Data Communication through HyperTerminal

The following Figures show the serial data transmission from teststation1 PC to teststation2 PC through RS232 interface. When the serial data is typed on HyperTerminal of master PC, it is transmitted through the project module 1 and received at the project module2, and then sent through the RS232 interface to HyperTerminal of slave PC. 


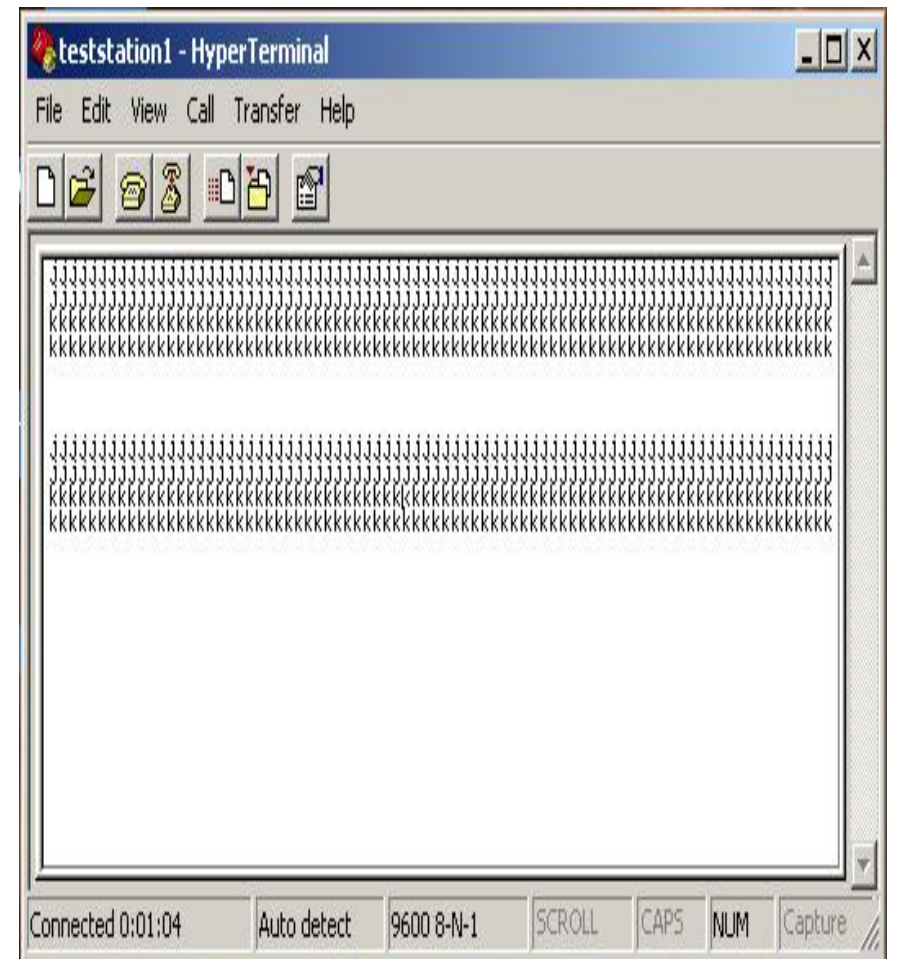

Fig. 5: Data at teststation1

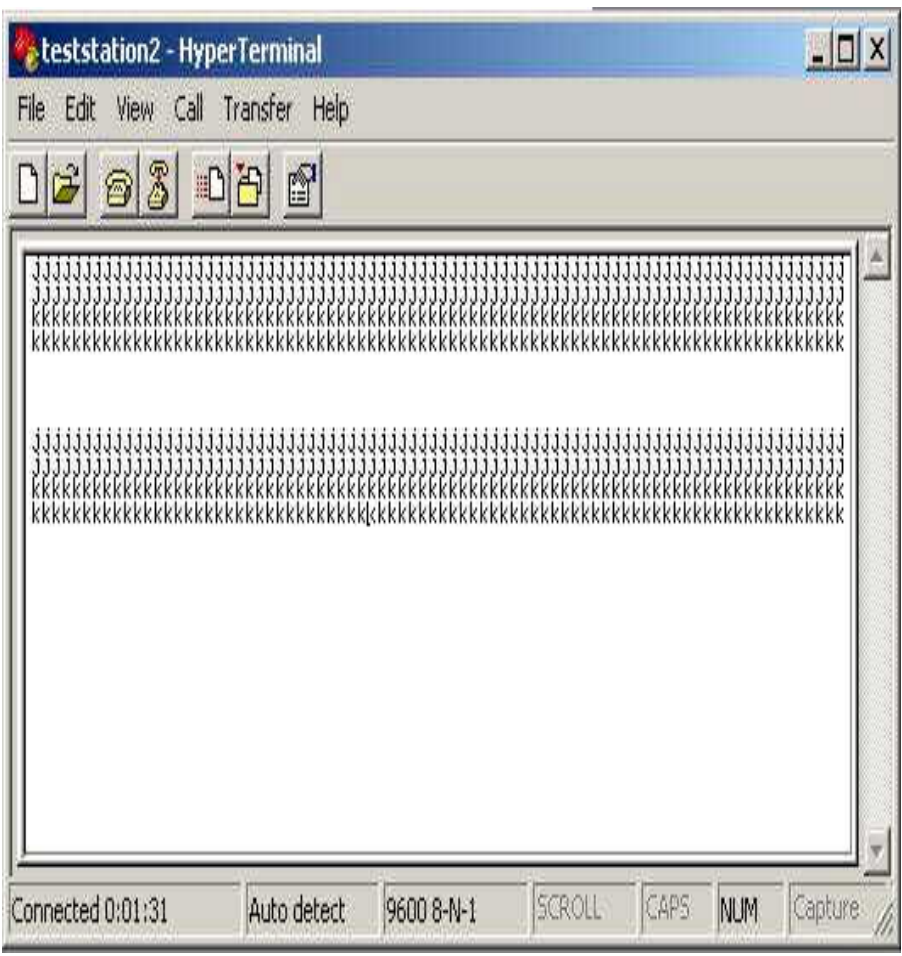

Fig. 6: Data at teststation2

\subsection{Snapshots of Test Results for Data and Clock at}

\section{the SPI Port}

The following Figure shows the continuous serial data at MOSI pin and clock at SCK pin of SPI port. Whenever the clock is present then only data is available at the MOSI pin. The ARM controller is programmed to transmit the continuous data through MOSI pin to RF IC.

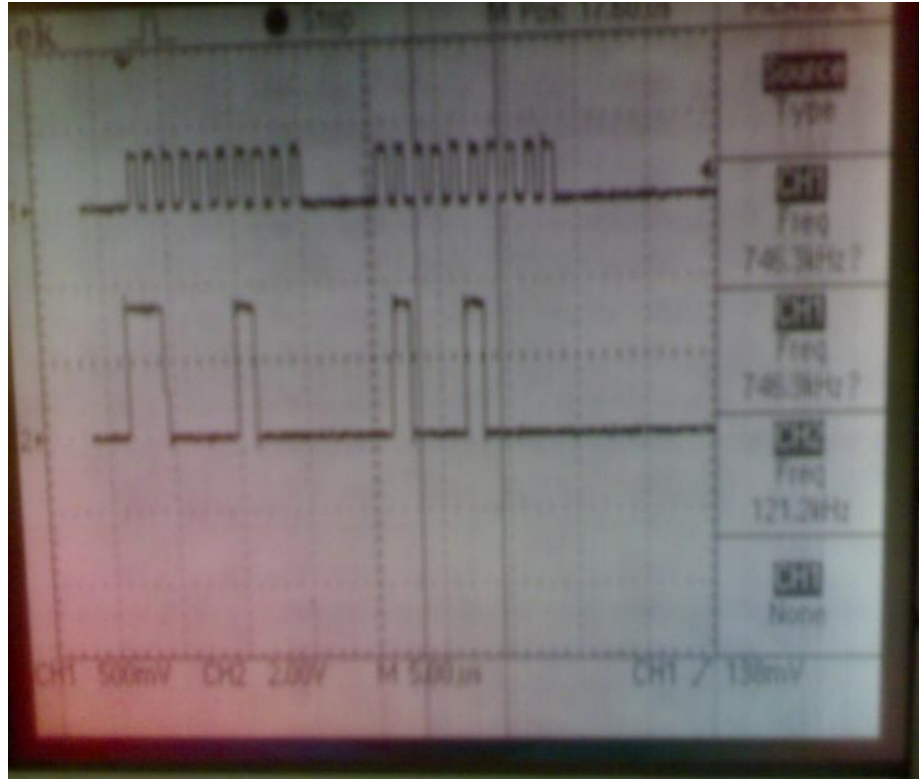

Fig.7: Data and clock at the SPI port

\subsection{Snapshots of Test results of Programs and its}

\section{Performance analyses}

The following figures show the Debugging of Main UART program and SPI program. The Fig. 6 shows the run time calculation and thus the performance is analyzed.

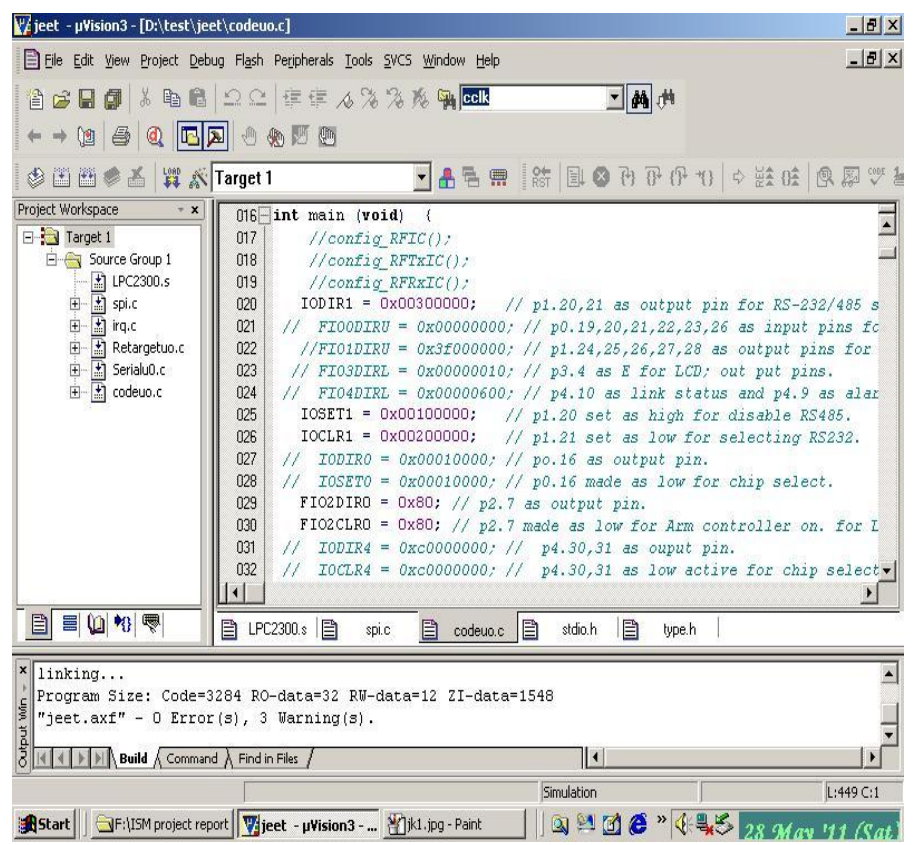

Fig. 8: Debugging Result of main Codeeu0.C 


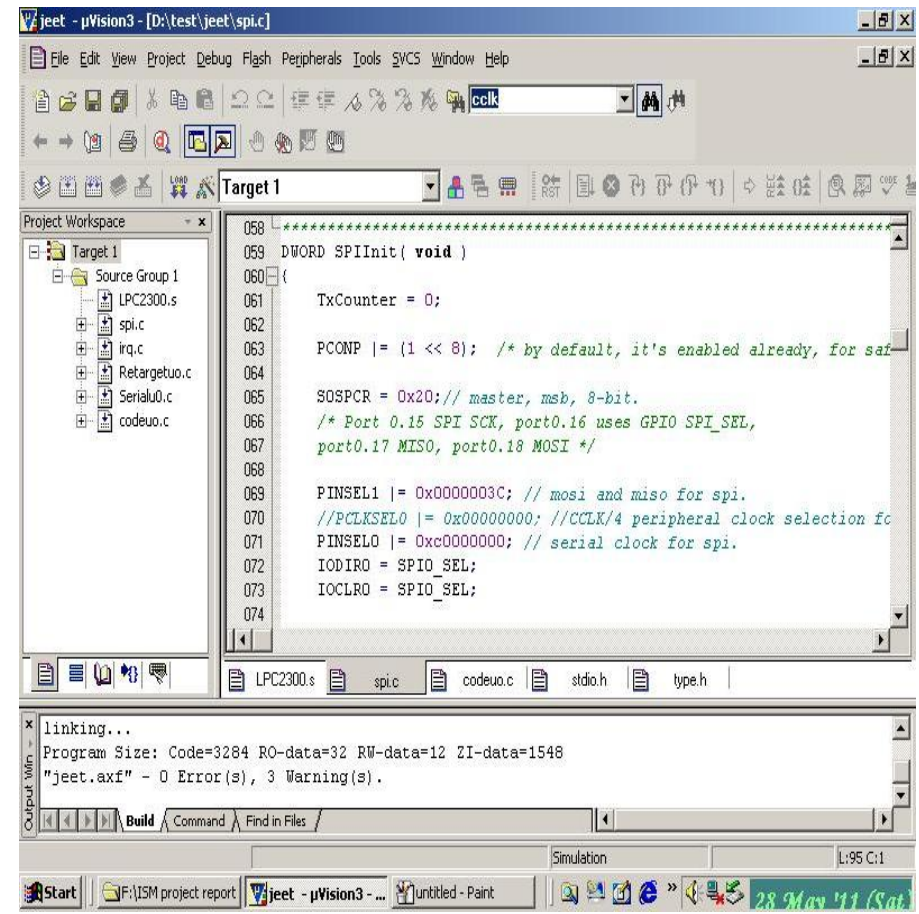

Fig. 9: Debugging Result of SPI.C Program

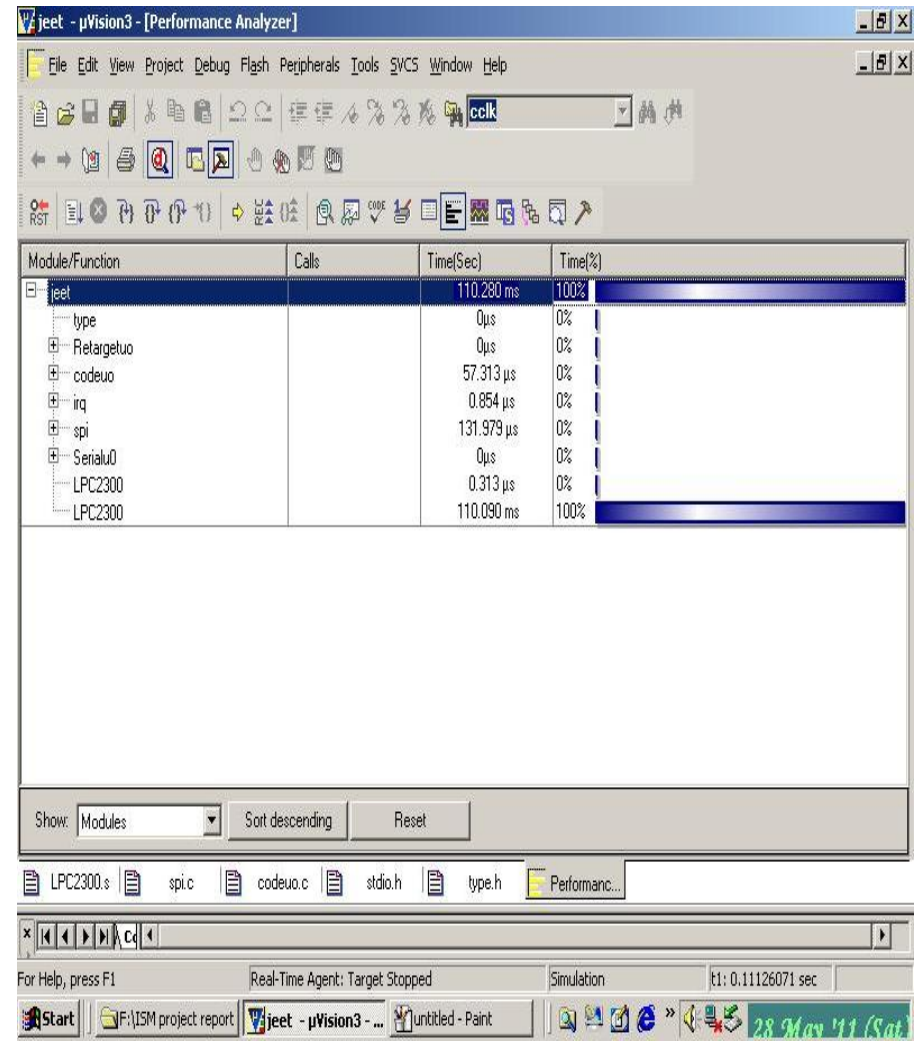

Fig. 10: Performance analyses of all Program

\section{CONCLUSIONS}

The Wireless ISM Transceiver has been successfully tested for the different ranges for the indoor application in terms power level of transmit and received signal strength in $\mathrm{dBm}$. It has been tested by hyper terminals of PCs between master module and slave module with RS-232 interface. The Output power of source module for indoor application is 0
$\mathrm{dBm}$. The Receiver sensitivity of the receiver module is observed at $-94 \mathrm{dBm}$.

\section{REFERENCES}

[1]. NXP (founded by Philips) - LPC23xx user manual.

[2]. MICROCHIP - MRF24J40 Data Sheet, IEEE 802.15.4 ${ }^{\mathrm{TM}} 2.4 \mathrm{GHz}$ RF Transceiver.

[3]. ZigBee ${ }^{\mathrm{TM}}$ Standards - IEEE 802.15.4

[4]. TI (Texas Instruments) - $2.4 \mathrm{GHz}$ PCB TI Antennas.

[5]. National Semiconductors - DP8384I PHYTER® 10/100 Mbps Data Sheet.

[6].Jan Kodas, Petrwwagner, "Wireless Transceiver for Control of mobile Embedded Devices" IEEE papers, VSBTechnical University of ostrava., 2010.

[7]. V. Srovnal Jr., Z. Machacek, “ Wireless Controller for mobile Robotics and Industrial Embedded devices", in Eight International Conference on Network, 2009.

[8]. Maher M.Abd- Elrazzak', member, "A modified EShaped microstrip Antenna for Ultra wideband wireless system”, IEEE paper, 2007. 